\title{
Comprehensive Analysis of Ad Hoc Network Routing Protocols
}

\author{
Bindeshwar Singh Kushwaha \\ Banaras Hindu University \\ Department of Computer Science \\ Institute of Science \\ Varanasi, India
}

\author{
Pramod Kumar Mishra \\ Banaras Hindu University \\ Department of Computer Science \\ Institute of Science \\ Varanasi, India
}

\begin{abstract}
In this paper, we simulate three ad hoc network routing protocols namely DSDV(Destination Sequenced Distance Vector), DSR(Dynamic Source Routing) and AODV(Ad Hoc On Demand Distance Vector ) and simulation carries out using network simulator version 2. Furthermore, end-to-end delay, normalized routing load, packet delivery ratio, throughput and jitter are evaluated as function of number of nodes and pause time.
\end{abstract}

\section{General Terms}

Ad Hoc Network, Wireless Network

\section{Keywords}

Ah Hoc Routing Protocols, Comparative Analysis, Delay, Throughput

\section{Introduction}

Wireless ad hoc network is a self-organized self-configured collection of nodes that communicate over wireless links without help of any base stations or an access point, and nodes work as both hosts and routers. Furthermore, an ad hoc network can be deployed quickly with minimal overhead and this nature makes it suitable for emergency usage such as in an earthquake hit, in a disaster area, or where building infrastructure is expensive and in a conference room where people need to share information. Moreover, an ad hoc network is also an option for the connectivity to Internet with the co-operation of people.

In addition, choosing a suitable protocol is very important to deploy an ad hoc network, and simulation analysis of protocols help to do this without using resources and with minimum effort, additionally, simulation analysis is also useful for understanding the behavior of the protocols and to know weakness for the further improvements.

\section{RELATED WORK}

Many researchers have worked on performance modeling and analysis of routing protocols. In [5], Broch and Maltz analyze ad hoc routing protocols using ns- 2 and they emphasize on physical layer and MAC layer issues. Furthermore, in [6] a comparative theoretical analysis is done by [Mehran Abolhasan][ Tadeusz Wyso cki][Eryk Dutkiewicz] in which convergence time, memory overhead, control overhead of proactive protocols, time complexity; communication complexity; route discovery; route maintenance of reactive routing protocols and hybrid protocols are analyzed. In [7] packet delivery fraction, average end to end delay, packet loss, routing overhead of AODV and DSR protocol are evaluated using ns2 simulator, [8] compares AODV and OLSR in different network scenarios. In [12], control overhead, packet delivery ratio, end to end delay and throughput are evaluated at the different pause time and in the different network sizes. In[13| different routing protocols are classified based on their reactive, proactive and hybrid nature, [15][14] evaluated performance of ad hoc routing protocols in different mobility models, and [16] assessed the protocols in horizontal and vertical scenarios. Rest of this paper is organized as follows. In section-[1] we discuss related work, in section-[2] we describe types of ad hoc network routing protocols. In section-[3][4][5] a brief introduction of simulated routing protocols. Further, section[6] gives overview of the environment.

In section[7] simulated results are discussed

Finally, in section-[8] we conclude the paper.

\section{AD HOC NETWORK ROUTING PROTOCOLS}

The ad hoc routing protocols can broadly be classified into two categories based on their characteristics i.e their root discovery process, keeping information always or whenever necessary.

\subsection{Proactive Routing Protocols}

In proactive routing protocols, every time nodes keep information up-to-date in their routing tables, and whenever a change is occurred in the network due to link broken, link failure and movement of a node nodes update their tables. Furthermore, the protocols impose heavy control overhead on the network, however, they minimize end-to-end delay.

Examples are DSDV[1], OLSR[4].

\subsection{Reactive Routing Protocols}

On the other hand, reactive routing protocols do not maintain information up-to-date like proactive routing protocols, they create the route whenever necessary. Moreover, the protocols minimize con- 
trol overhead at the cost of long delay in route discovery. Examples are AODV[2], DSR[3].

\section{DSDV}

DSDV[1](Destination Sequenced Distance Vector) is a proactive protocol, which has a routing table to keep information about all nodes which are in the network. The routing table entries are destination, next hop, metric, sequence number and flags.

In addition, it guarantees a loop free path due to a sequence number generated by the destinations. Furthermore, consistency of routing table is maintained by sharing information to neighbors, in two ways nodes share information full dump table and incremental dump, in full dump nodes share entire table to periodically, between two full dumps a node exchange incremental dumps when small changes is occurred in network such as sequence number or route change.

\section{DSR}

DSR [3] (Dynamic Source Routing) is a reactive protocol that uses source routing method i.e. it lists all the addresses of intermediate nodes in the packet's header of source node. Moreover, DSR maintains route cache in which it keeps previously learned route to the source node, and has an expiration period after that it is deleted. The protocol works in two phases. First, it initiates route discovery process by broadcasting route request packet throughout the network and when a node receives this packet it checks whether is it in route cache or not, it is in its route cache node discards and do not process, and if node itself is destination node it copies list of hopes to the source node into its route reply packet and sends to the source node. Further, if it is not target node and it does not have route entry for received packet it appends its address to route record and forward to the nodes which are within its transmission range. Second, route maintenance phase in which when an error occurs, for instance, link broken and movement of a node both nodes send root error packet to upstream nodes to notify there is error in the network, and after getting route error source node again initiates route discovery process.

\section{AODV}

AODV[2](Ad Hoc On Demand Distance Vector ) is a reactive routing protocol which is combination of DSDV[1] and DSR[3] (Dynamic Source Routing) routing protocols . AODV hires concept of destination sequence number from DSDV and route discovery and route maintenance concept from DSR. Protocol does not keep information about all nodes in its routing table in advance like its counterparts proactive routing protocols, nodes create a route to destination whenever necessary.

\section{SIMULATION ENVIRONMENT}

To carry out experiment we use ns-2[17] the simulation area we take 800 square meter, random way point mobility model is taken for movement of node which is very popular among researchers.

\begin{tabular}{|c|c|}
\hline Parameters & Value(s) \\
\hline Number of Nodes & $10,20, \ldots, 90,100$ \\
\hline Pause Time & $10,15, . ., 45,50$ \\
\hline Simulation Area & 800 meter square \\
\hline Propagation Model & Two ray ground \\
\hline Mobility Model & Random Way Point \\
\hline Antenna Model & Omni direction \\
\hline MAC Layer & $802.11 \mathrm{~b}$ \\
\hline Routing Protocols & DSDV, DSR, AODV \\
\hline Transport Layer & UDP \\
\hline Application Layer & CBR \\
\hline Packet Size & 512 bits \\
\hline Application Data Rate & 500 kilo bits/second \\
\hline
\end{tabular}

Additionally, we simulate three routing protocols DSDV, AODV and DSR with we vary parameter number of nodes when pause time is 10 second (a constant), and we increase pause time and keep number of nodes 100 (a constant). CBR traffic pattern is considered as application data over UDP transport layer protocol and 802.11b model is chosen for physical layer and data link layer.

\section{PERFORMANCE ANALYSIS}

The performance metrics we have taken from RFC 2501[9][10] in which quantitative and qualitative metrics are defined and jitter metrics from RFC 5148[11] in which jitter is explained.

\subsection{End To End Delay}

End to End Delay is defined as time (T) taken by a packet to travel from the application layer of source node to the application layer of destination node.

$$
\text { EED }=\text { Pr }- \text { Pg }
$$

\section{Pr Time at reception of packet}

\section{$\mathrm{Pg}$ Time at generation of packet}

DSDV has less EED than AODV and DSR as it maintains information every time whenever a change is occurred in the network, and when a node needs to send data packet to destination there is no need to discover root. Although, AODV and DSR do not maintain root to all nodes and when a node wants to communicate with destination node both protocols initiate route discovery protocol that causes delay.

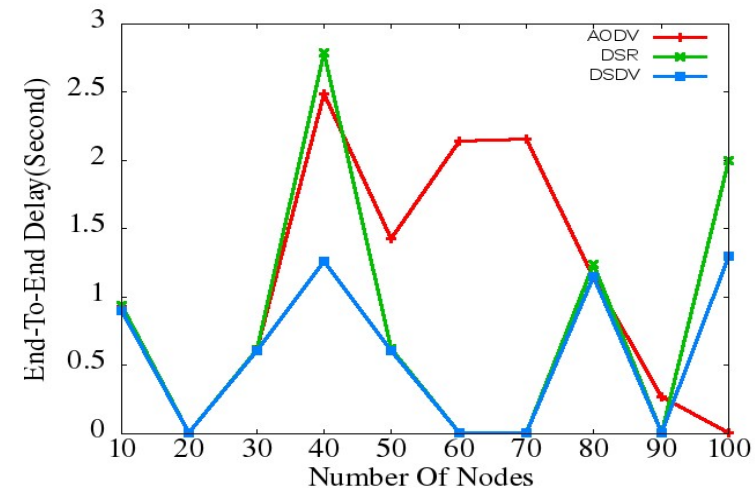

Fig. 1: Number of Nodes Vs End-To-End Delay

In this case, it can seen that from the figure[1] when number of 
nodes are increased end to end delay is also increased but it is less compared to AODV and DSDV.

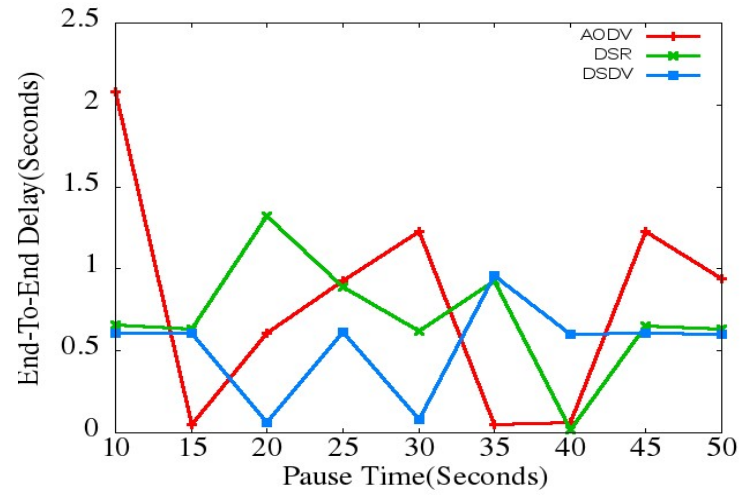

Fig. 2: Pause Time Vs End-To-End Delay

In figure [2] when pause time increases nodes become stable, AODV and DSR also performs like DSDV.

\subsection{Normalized Routing Load}

Normalized Routing Load(NRL) is defined as the ratio of control packets to data packets, and it characterizes the protocol overhead to send data from a source node to its respective destination.

Normalized Routing Load can be expressed as.

$$
N R L=\frac{P c}{P d}
$$

\section{Where \\ Pc Number of Control Packets \\ Pr Number of Data Packets}

In figure [2], it can be figured out that DSDV has higher routing overhead compare to DSR and AODV. Since DSDV updates its routing table every time whenever a change is occurred in the network, even though, when nothing is going on in the network it emits control packets. In both cases, NRL as the function of number of nodes and NRL as the function of pause time DSDV has highest routing overhead.

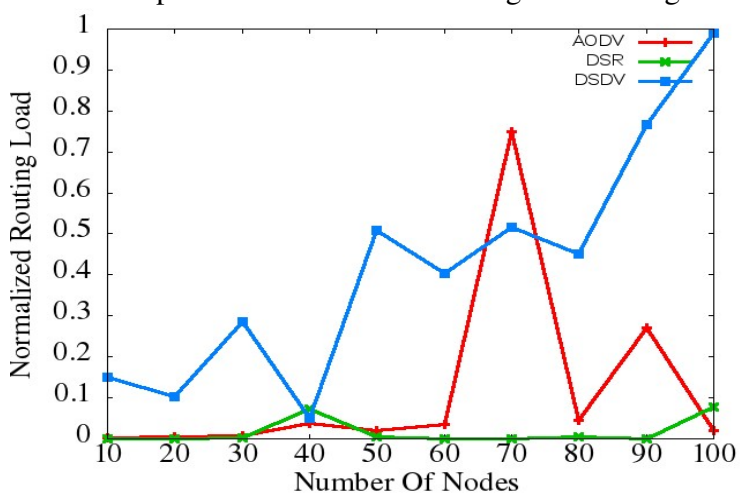

Fig. 3: Number of Nodes Vs NRL

So, DSDV has the highest routing overhead among three protocols. DSR has the lowest NRL because it emits control packets very less compare to AODV and DSDV.

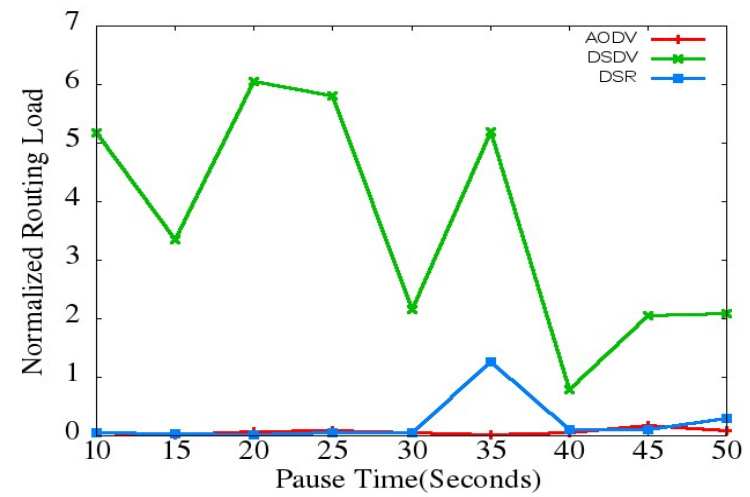

Fig. 4: Pause Time Vs Normalized Routing Load

\subsection{Packet Delivery Ratio}

Packet delivery fraction is the ratio of packets received by destinations and sent by sources across the network.

$$
P D R=\frac{P r}{P s}
$$

\section{Where \\ Pr Total Number of Packets Received. \\ Ps Total Number of Packets Sent.}

As DSDV maintains routing table and also DSR maintains route cache, and they keep information longer compare to AODV and less number of packets loss. As a result, the packet delivery ratio of DSDV and DSR is higher compared to AODV.

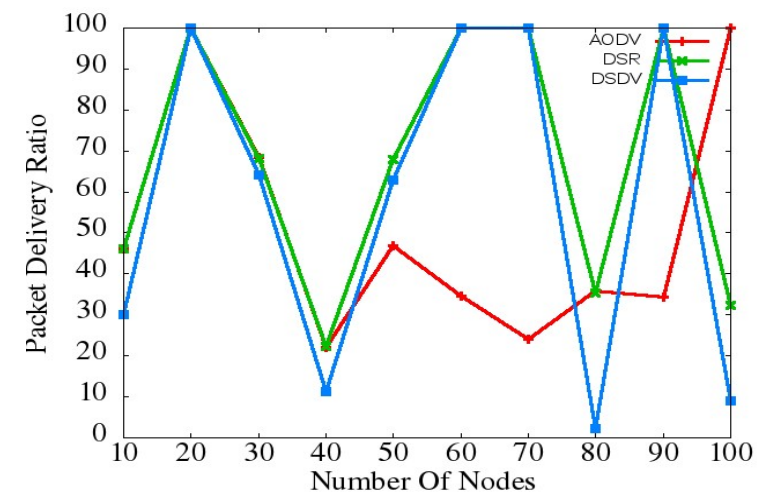

Fig. 5: Number of Nodes Vs PDR

From figure[6], it can be observed when nodes become stable 
AODV performance is higher compared to AODV and DSDV.

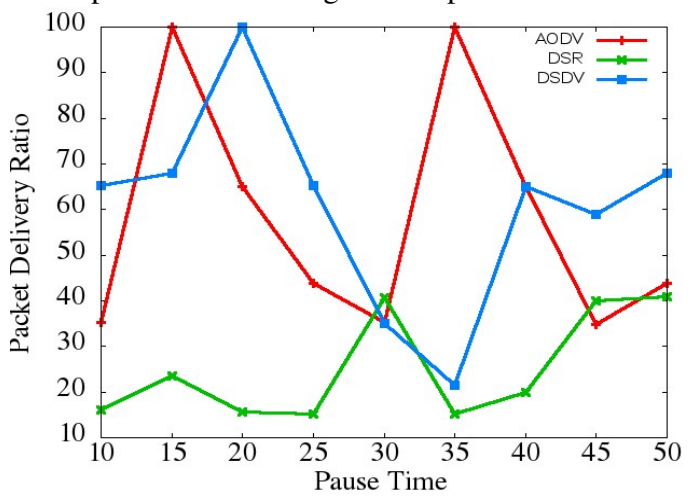

Fig. 6: Pause Time Vs PDF

\subsection{Jitter}

Jitter is defined as the difference in delay between two successive packets related to the same flow.

$$
\text { Jitter }=\operatorname{Delay}(P n)-\operatorname{Delay}(P n-1)
$$

Variation in delay (Jitter) is occurred when different delays are experienced by packets related to the same flow, it may happen at node queue when a packet arrives at input queue wait for some time and after that node forwards through outgoing queue at different delays while packets belong to the same flow. Figures demonstrate that DSDV has less delay compare to DSR and AODV. Since DSDV has less end to end delay and, as a result, it performs better in case of jitter.

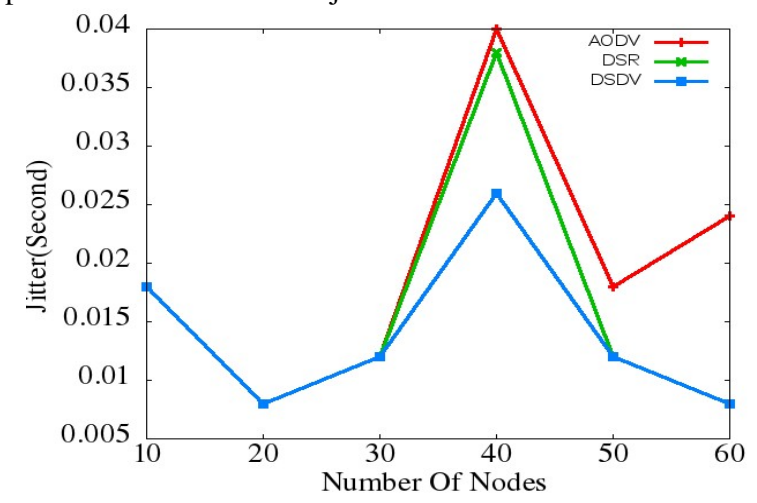

Fig. 7: Number of Nodes Vs Jitter

In the first case, jitter as function of number of nodes, DSDV performance is better but in the second case when pause time increase all three protocols perform equivalently.

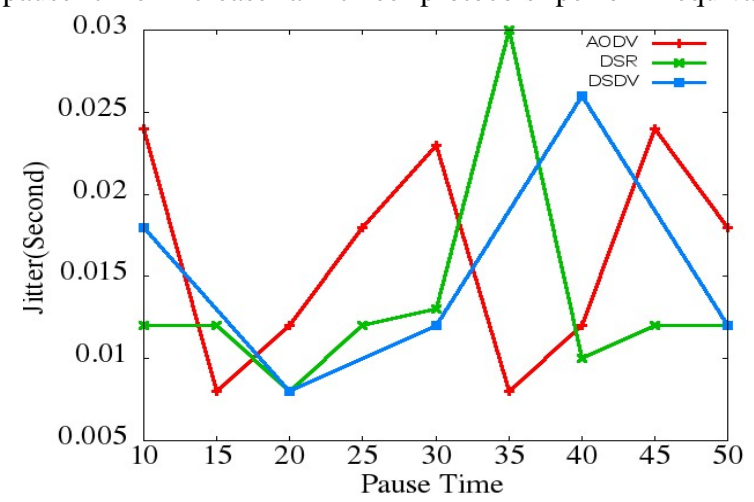

Fig. 8: Pause Time Vs Jitter

\subsection{Throughput}

The network throughput is referred to as the average number of bits received by destination nodes in one second.

$$
\text { Throughput }=\frac{8 * T B}{T L B R-T F B S}
$$

\section{Where \\ TB \\ TFBS \\ Total Number of Bytes \\ Time at First Bit Sent \\ TLBR Time at Last Bit Received}

As DSDV updates routing table every time and DSR maintains route cache, probability of packet loss is less than AODV, for this reason, both protocols have higher throughput than AODV, when number of nodes are increased.

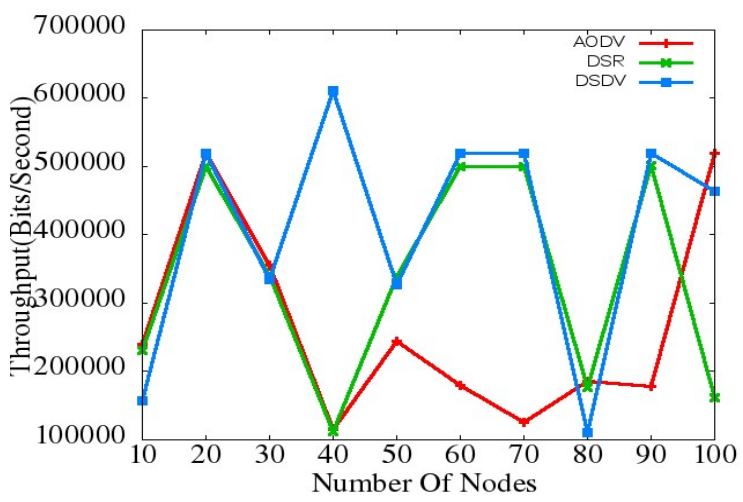

Fig. 9: Number of Nodes Vs Throughput

From figure[10] it can be observed that when pause time increases nodes become stable for longer time and packet loss rate slows 
down, in this case all three protocols give similar performance.

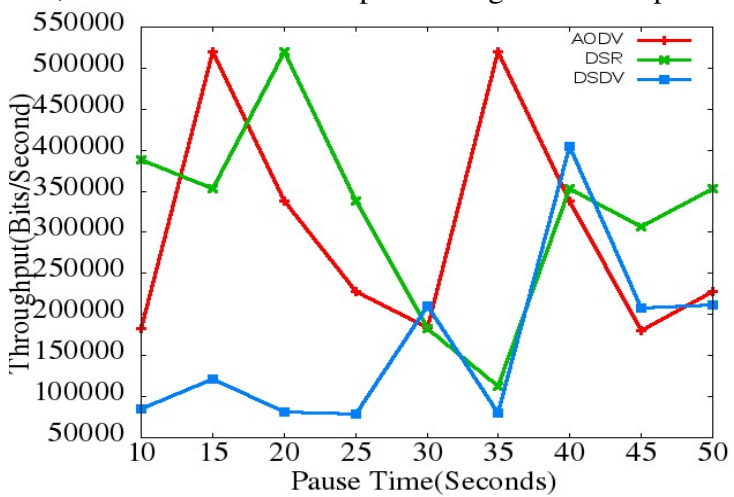

Fig. 10: Pause Time Vs Throughput

\section{CONCLUSION AND FUTURE WORK}

From performance analysis of ad hoc network routing protocols it can be concluded that DSDV has less end to end delay but it imposes heavy burden on the network i.e routing overhead. However, AODV and DSR have less routing overhead than DSDV but they causes long delay in route discovery. Furthermore, in case of jitter, AODV has higher jitter compare to DSDV and DSR. In addition, cross layer design for performance enhancement in ad hoc network has drawn more and more attention of researchers, and we have planned to work on this issue. Additionally, security features, Quality of Service (QoS), mobility management are very attractive areas of research in in the field of ad hoc network and many researchers are working on this issues. Moreover, applications on ad hoc networks are very appealing, such peer to peer network for file sharing among community members, VOIP, for disaster areas, video streaming for rescue operation are hot areas of research.

\section{REFERENCES}

[1] Charles E. Perkins, Pravin Bhagwat, Highly Dynamic Destination-Sequenced Distance Vector Routing for Mobile Computers, SIGCOMM 1994 ACM pages 234-244.

[2] C. Perkins, E. Belding Royer, S.Das, Ad hoc On- Demand Distance Vector (AODV) Routing, Internet RFC 2003, pages 1-38.

[3] D. Johnson, D. Maltz, J.Broach, The Dynamic Source Routing protocol multi hop wireless ad hoc networks, 2001, pages 1-25.

[4] T. Clausen, P. Jacquet, Optimized Link State Routing (OLSR) Protocol, IETF RFC3626, 2003.

[5] J.Broch, D.Maltz, D.Johnson et al, A performance comparison of multi hop wireless ad hoc network routing protocols, 1998, 85-87.

[6] M. Abolhasan, T. Wysocki, E.Dutkiewicz, A review of routing protocols for mobile ad hoc networks, 2004, pages 1-22.

[7] Sunil Taneja, Ashwani Kush, Evaluation of Normalised Routing Load for MANET, Springer Verlag Berlin Heidelberg, 2011.

[8] Aleksandr Huhtonen, Comparing AODV and OLSR Routing Protocols, HUT T-110-551, Seminar On Internetworking.

[9] S. Corson, J. Macker, Routing Protocol Performance Issues and Evaluation Considerations, RFC 2501, 1999.

[10] Mdhavi W. Subbarao, Ad Hoc Networking Critical Features and Performance Metrics, Wireless Communications Technology Group, NIST, October 7, 1999.
[11] T. Clausen, C. Dearlove, B. Adamson, Jitter Considerations in MANET, RFC[5148], 2008.

[12] S. Mohapatra, P.Kanungo, Performance analysis of AODV, DSR, OLSR, and DSDV Routing Protocols Using NS2 simulator, International Conference on Communication Technology.

[13] Elizabeth M. Royer, A Review of Current Routing Protocols for Ad Hoc Mobile Wireless Networks, IEEE Personal Communications, 1999.

[14] Ricardo de Oliveira Schmidt, Marco Antnio Sandini Trentin, MANETs Routing Protocols Evaluation in a Scenario with High Mobility.

[15] Fahim Maan, Nauman Mazhar, MANET Routing Protocols vs Mobility Models: A Performance Evaluation , IEEE ICUFN 2011.

[16] Masahiro Hiyama, Elis Kulla, Makoto Ikeda, Leonard Barolli and Arjan Durres, Comparison Evaluation of Horizontal and Vertical Scenarios for Delay and Jitter Metrics Using a MANET Testbed, 2011 International Conference on Emerging Intelligent Data and Web Technologies.

[17] Network Simulator Version 2 Documentation "http://www.isi.edu/nsnam/ns/". 